\title{
Research Paper \\ A Comparative Study on Emotional Regulation in Males With Internet Addiction, Food Addiction, Opioid Dependence and Normal Peers
}

\author{
${ }^{*}$ Shirzad Babaei ${ }^{1} \oplus$, Ali Asghar Asgharnejad Farid ${ }^{1} \oplus$, Fahimeh Fathali Lavasani ${ }^{1}\left(\mathbb{C}\right.$, Behrooz Birashk $^{1} \oplus$
}

1. Department of Clinical Psychology, School of Behavioral Sciences and Mental Health (Tehran Institute of Psychiatry), Iran University of Medical Sciences, Tehran, Iran.

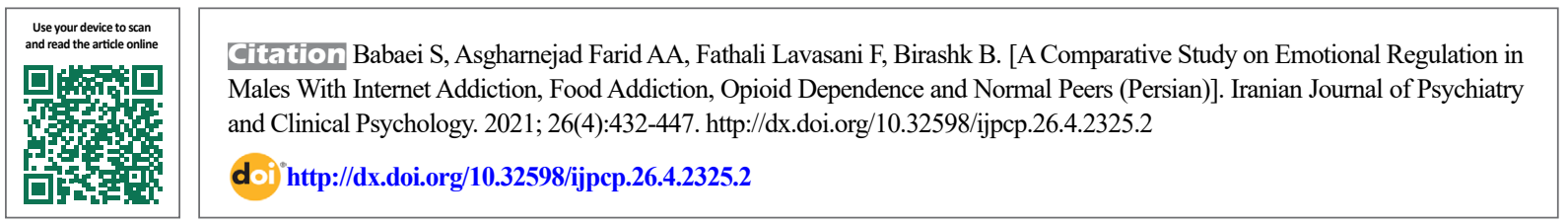

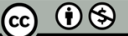

Received: 20 Aug 2019 Accepted: 05 Jul 2020 Available Online: 01 Jan 2021

Keywords: Emotional regulation Opioid dependence, Internet addiction, Food addiction

\begin{abstract}
A B STRACT
Objectives Difficulty in emotional regulation is an important component in the pathology of different types of addiction and its evaluation can be helpful in understanding and treating these difficulties. The purpose of this study is to evaluate emotional regulation in people with opioid dependence (heroin and opium), food addiction, and internet addiction compared to normal people.

Methods This is a causal- comparative study. Participants were 120 eligible male addicts selected using a purposive sampling method and divided into four groups of 30 including drug addiction, food addiction, internet addiction, and control. Semi-structured clinical interview for DSM-IV axis I disorders, Yale Food Addiction Scale, and Young's Internet Addiction Test were used to diagnose the addictions. Then the Difficulties in Emotion Regulation Scale (DERS) was completed. Multivariate Analysis of Variance (MANOVA) was used to analyze the collected data.

Results Overall DERS score and the score of its subscales were higher in all three addiction groups compared to the control group, and this difference was statistically significant $(P<0.05)$. There was no significant difference between the three addiction groups in subscales of "unwillingness to accept certain emotional responses" and "difficulty engaging in goal-directed behavior", but a significant difference between the three addiction groups was observed in subscales of "difficulty controlling impulse", "lack of emotional awareness", "lack of access to strategies", "lack of emotional clarity", and overall DERS score $(P<0.05)$. Conclusion Men with opioid dependence, internet addiction and food addiction have more difficulties in emotional regulation compared to their normal peers. Emotional regulation ability in men with internet addiction is greater than in those with food and opioid addictions.
\end{abstract}

\section{Extended Abstract}

\section{Introduction}

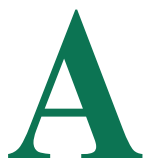

ddiction is one of the most important social problems in which social and psychological factors, on the one hand, and biological and pharmacological factors on the other hand are involved. Recently, the term "addiction", in addition to substance abuse, has been used to refer to a wide range of repetitive behaviors such as the Internet use, eating, gambling, video gaming, physical exercises, and sexual intercourse. Recent studies have shown that addiction to food, internet, and substances are associated with emotional problems. Emotional dysregulation occurs when one is unable to use the necessary skills to regulate negative emotions or emotional tension. Since various studies have reported similarities between substance abuse and food addiction, and the Internet addiction as a behavioral

\section{* Corresponding Author:}

\section{Shirzad Babaei, PhD.}

Address: Department of Clinical Psychology, School of Behavioral Sciences and Mental Health (Tehran Institute of Psychiatry), Iran University of Medical Sciences, Tehran, Iran.

Tel: +98 (918) 2884151

E-mail: shirzadbabaei@gmail.com 
addiction and similar to substance abuse and food addiction, also cause problems with emotional regulation, this study aims to investigate and compare emotional regulation as a common component in men with substance abuse, food addiction, and Internet addiction.

\section{Methods}

This is a causal-comparative study. The study population consists of all men with opioid dependence (heroin and opium) referred to Tehran Municipality residential centers, all male students with internet addiction in Tehran University of Medical Sciences, and all men with food addiction referred to obesity clinics of Imam Khomeini Hospital in Tehran, Iran. The samples were selected using a purposive sampling and a convenience sampling techniques, and divided into four groups of food addiction $(\mathrm{n}=30)$, Internet addiction $(\mathrm{n}=30)$, drug addiction $(\mathrm{n}=30)$, and control $(\mathrm{n}=30)$ groups. The inclusion criteria were: Having informed consent, male gender, age 20-40 years, no psychotic and eating disorders, and the absence of a diagnosis of binge eating disorder (to avoid overlapping with food addiction), having at least a high school diploma up to a bachelor's degree, and having symptoms related to each addiction. To diagnose addictions, a Semi-structured Clinical Interview for DSM-IV axis I (SCID-I) disorders, Yale Food Addiction Scale (YFAS), and Internet Addiction Test (IAT) were used at baseline. Then, subjects completed the Difficulties in Emotion Regulation Scale (DERS). Collected data were analyzed in SPSS V.16 software using Chi-square test to determine examine the difference between four groups in terms of education, one-way ANOVA to determine difference between groups in terms of age, and MANOVA to compare the mean score of variables in the study groups (Table 1).

\section{Results}

The results showed a significant difference between the four groups in terms of all DERS subscales and its overall score $(\mathrm{P}<0.05)$, which were higher in all three addiction

Table 1. Tukey's test for multiple comparison of study groups (Dependent variable: DERS)

\begin{tabular}{|c|c|c|c|c|}
\hline Variable & Group (I) & Group (J) & Mean Difference (Std. Error) & Sig. \\
\hline \multirow{6}{*}{$\begin{array}{l}\text { Unwillingness to accept certain emo- } \\
\text { tional responses }\end{array}$} & \multirow{3}{*}{ Drug addiction } & Food addiction & $-0.83(0.88)$ & 0.78 \\
\hline & & Internet addiction & $-0.10(0.88)$ & 0.99 \\
\hline & & Control & $3.50(0.88)$ & 0.001 \\
\hline & \multirow{2}{*}{ Food addiction } & Internet addiction & $0.73(0.88)$ & 0.84 \\
\hline & & Control & $4.33(0.88)$ & 0.001 \\
\hline & Internet addiction & Control & $3.60(0.88)$ & 0.001 \\
\hline \multirow{7}{*}{$\begin{array}{l}\text { Difficulty engaging in goal-directed } \\
\text { behavior }\end{array}$} & \multirow{3}{*}{ Drug addiction } & Food addiction & $0.33(0.93)$ & 0.98 \\
\hline & & Internet addiction & $1.70(0.93$ & 0.26 \\
\hline & & Control & $5.80(0.93)$ & 0.001 \\
\hline & \multirow{3}{*}{ Food addiction } & Internet addiction & $1.36(0.93)$ & 0.46 \\
\hline & & & & \\
\hline & & Control & $5.46(0.93)$ & 0.001 \\
\hline & Internet addiction & Control & $4.10(0.93)$ & 0.001 \\
\hline \multirow{7}{*}{ Difficulty controlling } & \multirow{3}{*}{ Drug addiction } & Food addiction & $1.43(1.01)$ & 0.49 \\
\hline & & Internet addiction & $5.73(1.01)$ & 0.001 \\
\hline & & Control & $11.46(1.01)$ & 0.001 \\
\hline & \multirow{3}{*}{ Food addiction } & Internet addiction & $4.30(1.01)$ & 0.001 \\
\hline & & & & \\
\hline & & Control & $10.03(1.01)$ & 0.001 \\
\hline & Internet addiction & Control & $5.73(1.01)$ & 0.001 \\
\hline
\end{tabular}




\begin{tabular}{|c|c|c|c|c|}
\hline Variable & Group (I) & Group (J) & Mean Difference (Std. Error) & Sig. \\
\hline \multirow{7}{*}{ Lack of emotional a } & & Food addiction & $-0.06(1.03)$ & 0.01 \\
\hline & Drug addiction & Internet addiction & $3.63(1.03)$ & 0.003 \\
\hline & & Control & $6.90(1.03)$ & 0.001 \\
\hline & & Internet addiction & $3.70(1.03)$ & 0.003 \\
\hline & Food addiction & & & \\
\hline & & Control & $6.96(1.03)$ & 0.001 \\
\hline & Internet addiction & Control & $3.26(1.03)$ & 0.01 \\
\hline \multirow{7}{*}{ Lack of access to st } & & Food addiction & $-2.13(1.03)$ & 0.17 \\
\hline & Drug addiction & Internet addiction & $3.63(1.03)$ & 0.004 \\
\hline & & Control & $11.10(1.03)$ & 0.001 \\
\hline & & Intornot addiction & $576(102)$ & 0001 \\
\hline & Food addiction & & & \\
\hline & & Control & $13.23(1.03)$ & 0.001 \\
\hline & Internet addiction & Control & $7.46(1.03)$ & 0.001 \\
\hline \multirow{16}{*}{ Lack of emotiona } & & Food addiction & $-0.60(0.91)$ & 0.91 \\
\hline & Drug addiction & Internet addiction & $3.83(0.91)$ & 0.001 \\
\hline & & Control & $4.96(0.91)$ & 0.001 \\
\hline & & & & \\
\hline & & Internet addiction & $4.43(0.91)$ & 0.001 \\
\hline & Food addiction & & & \\
\hline & & Control & $5.56(0.91)$ & 0.001 \\
\hline & Internet addiction & Control & $5.13(0.91)$ & 0.001 \\
\hline & & Food addiction & $-1.83(4.32)$ & 0.97 \\
\hline & Drug addiction & Internet addiction & $18.43(4.32)$ & 0.001 \\
\hline & & Control & $44.16(4.32)$ & 0.001 \\
\hline & & & & \\
\hline & & Internet addiction & $20.26(4.32)$ & 0.001 \\
\hline & Food addiction & & & \\
\hline & & Control & $46.00(4.32)$ & 0.001 \\
\hline & Internet addiction & Control & $25.73(4.32)$ & 0.001 \\
\hline
\end{tabular}

groups compared to controls. The total DERS score was higher in the drug addiction group compared to the Internet addiction group, and also was higher in the food addiction group compared to those with internet addiction, but it was almost the same between drug addiction and food addiction groups. There was no significant difference in subscales of "unwillingness to accept certain emotional responses" and "difficulty engaging in goal-directed behavior" between the three addiction groups, but a significant difference was reported between them in these subscales compared to the control group $(\mathrm{P}=0.01)$. Moreover, there was a significant dif- ference between the three addiction groups compared to the control group in subscale of "difficulty controlling impulse" $(\mathrm{P}=0.01)$; however, there was no significant difference between the drug addiction and food addiction groups. The difficulty controlling impulse was higher in the drug addiction group than in the Internet addicted group, and higher in the food addiction group than in the internet addiction group. The scores of subscales "lack of emotional awareness", "lack of access to strategies", and "lack of emotional clarity" were also higher in all three addiction groups compared to the control group. Their scores were higher in those with opi- 
oid dependence compared to those with Internet addiction, and were higher in subjects with food addiction compared to those with Internet addiction, but their scores were almost the same between drug addiction and food addiction groups.

\section{Discussion}

The results of our study showed that in opioid dependent groups, people with food addiction and those with Internet addiction had more difficulty in emotional regulation in comparison with the normal people. The difficulty level were almost the same between opioid and food addiction groups and was even lower in compared to those with Internet addiction. According to the results of this study and other studies, it seems that people with food addiction overeat and consume more foods as a defensive mechanism against negative emotions and low self-esteem. This mechanism is similar to that of drug addicts, which highlights the role of depression, anxiety, negative mood, and difficulty in emotional regulation as stimuli for substance use or food consumption. This means that they use maladaptive eating behaviors as a form of escaping from negative emotions, and biased towards food and substance during emotional arousals. Internet addiction is very similar to food addiction and substance abuse, but there is also an addiction to having a behavior; in this study, the higher difficulty in emotional regulation in two food and drug addictions compared to the Internet addiction can be due to a more direct effect of food and opioid on brain systems, as well as the vital role of food intake in survival compared to the mediating role of excessive Internet use and addiction. The similar characteristics of these three types of addiction reported in various studies have made them comparable.

In general, the results of this study are consistent with the self-medication theory of Khantzian. From this perspective, addictive behaviors derive from intolerable and painful emotions (e.g. depression, anxiety, shame, anger or feeling helpless), and substance use is an attempt to compensate the ego's inability to defend against these emotions. This theory is in line with clinical observations and studies that emphasize the important role of human mental suffering in addictive disorders. According to this theory, different individuals are accidentally begin to use substances and methods to reduce their discomfort and stress based on their specific psychological problems, and continue to use them depending on which substance, medication or behavior is effective in reducing their discomfort and stress. This puts the person into an dysfunctional and defective cycle.

\section{Ethical Considerations \\ Compliance with ethical guidelines}

In this study, a written informed consent was obtained from the participants, and their information kept confidential. An ethical approved was obtained from the Research Ethics Committee of the Iran University of Medical Sciences (Code: IUMS.REC.1394.9111556013).

\section{Funding}

This study was extracted from the MA. thesis of first author, Department of Clinical Psychology, School of Behavioral Sciences and Mental Health (Tehran Institute of Psychiatry), Iran University of Medical Sciences, Tehran. Also, It was conducted in cooperation with the Research Center and School of Behavioral Sciences \& Mental Health at Iran University of Medical Sciences, and School of Medicine of the Tehran University of Medical Sciences.

\section{Authors contributions}

Conceptualization and methodology and writing - original draft and writing - review \& editing: Shirzad Babaei; Investigation, funding acquisition, resources and supervision: All author.

\section{Conflicts of interest}

The authors declare no conflict of interest. 
This Page Intentionally Left Blank 


\title{
مقايسه نظمبخشى هيجانى در افراد مبتلا به اعتياد اينترنتى، غذايى، مواد افيونى و تروه كنترل
}

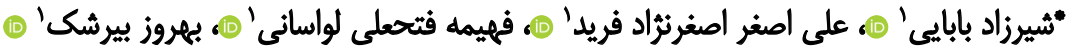 \\ ا. كروه روانشئاسى بالينى، دانشكده علوم رفتارى و سلامت روان (انستيتو روانيزشكى تهران)، انشكاه علوميزشكى ايران، تهران، ايران.
}

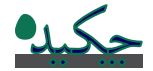

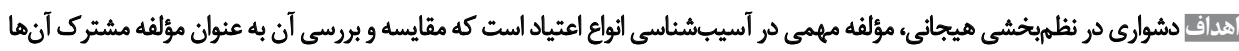

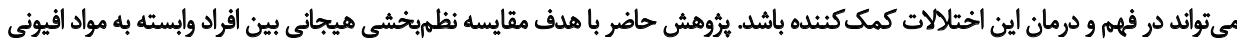

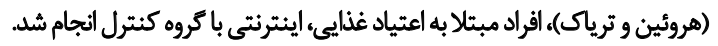

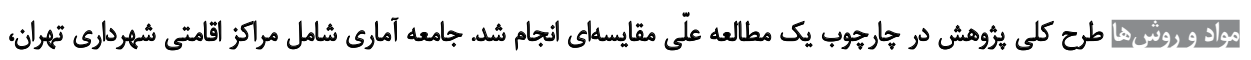

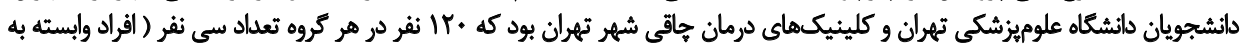

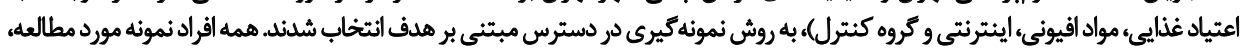

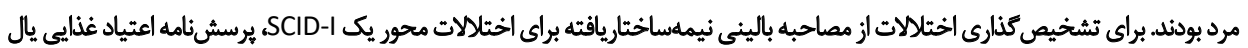

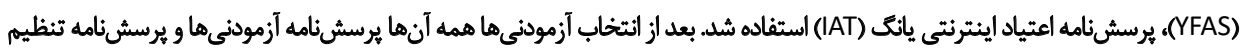

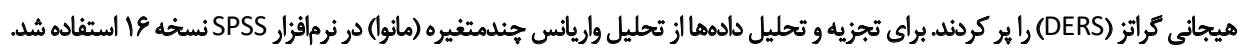

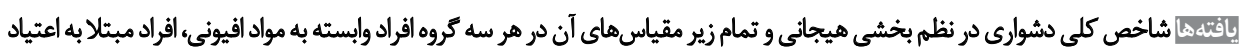

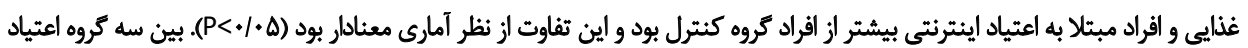

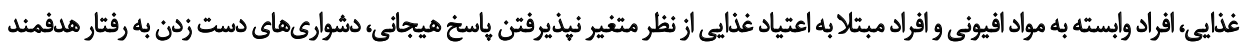

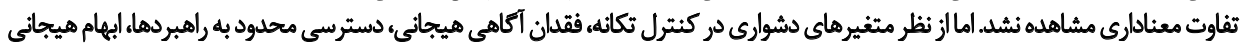

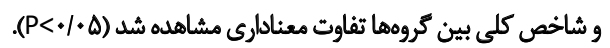

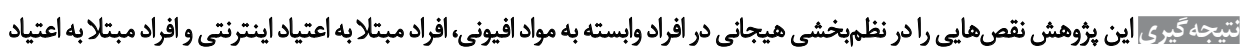

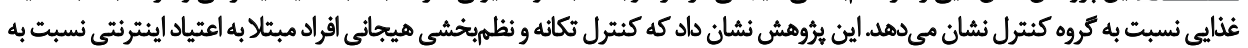

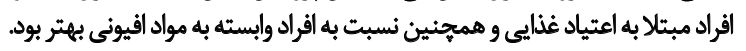

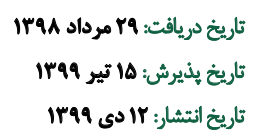

كليدواروها:

نظمبخشى هيجانى،

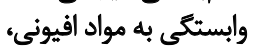
اعتياد اينترنتي، اعتياد

غذايى
وابستَّى' به مواد يك الثوى غيرانطباقى مصرف مواد است كه

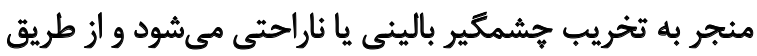

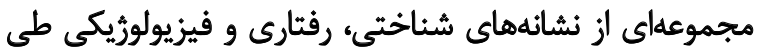

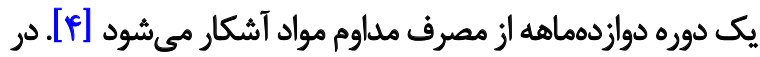

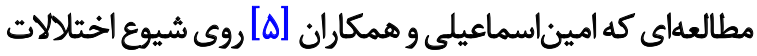

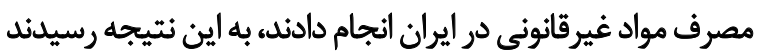

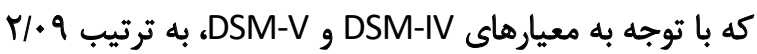

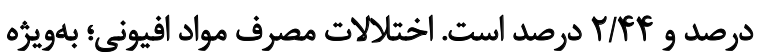
ترياك شايعترين اختلال مصرف بوده است اختالات دصرف موال

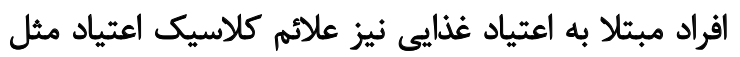

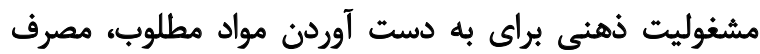

مقدمه

اعتياد يكى از مهمثرين مشكلات اجتماعى است كه در ايجاد آن

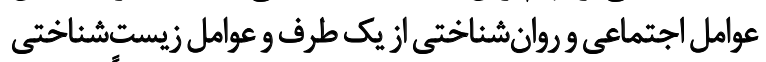

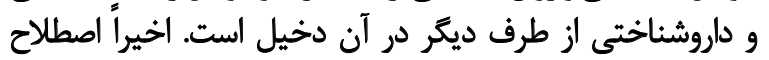

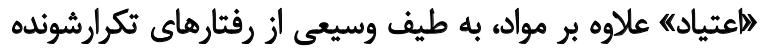

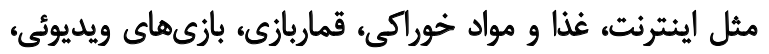

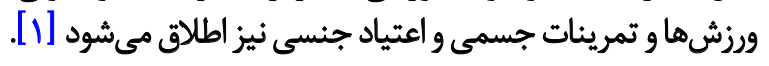

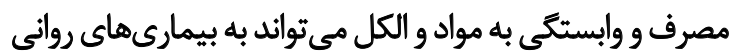

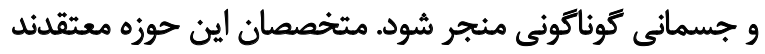

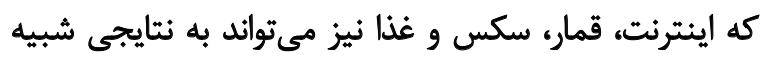

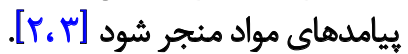


مكانيسمهاى مقابلهاى در رابطه هيجانات تنشزاى مرتبط با

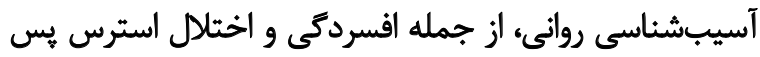

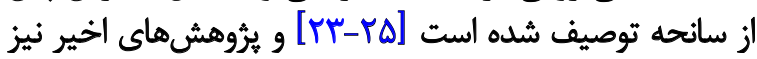

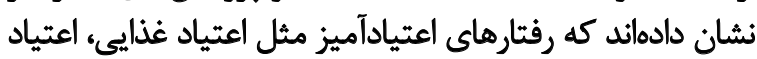

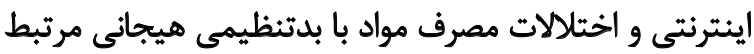

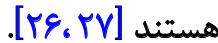

بدتنظيمى هيجانى زمانى اتفاق ميىافتد كه فرد نمى تونواند

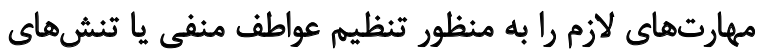

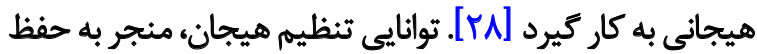

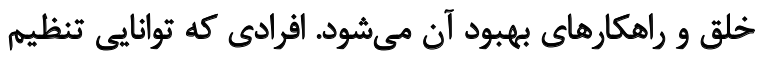

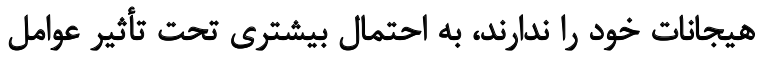

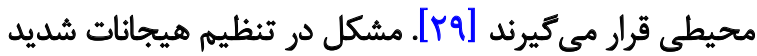

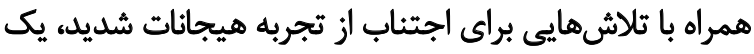

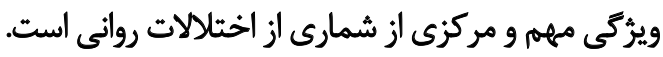
دشوارى در تنظيم هيجانى و تجربهاجتناب به عنوان فاكتورهاى

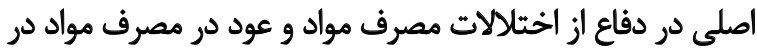

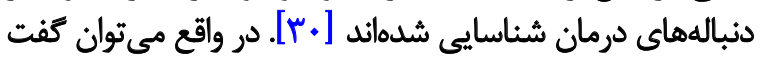

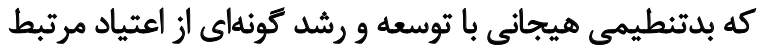

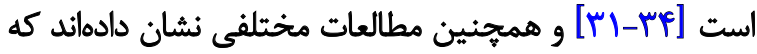

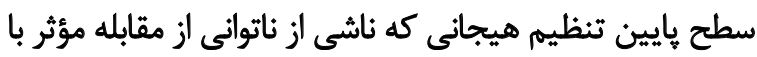

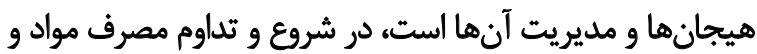
وابستكى به آن نقش داريت انهاد إها].

برخى يُروهشكران معتقدند كه مقايسه اعتياد به مواد و داروها،

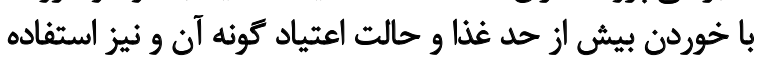

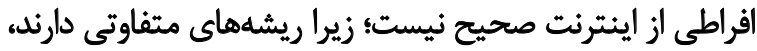

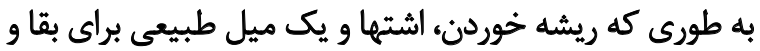

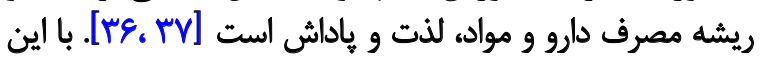
حال در يُوهشهاى اخير انجامشده روى موش اشها، نشان دادي

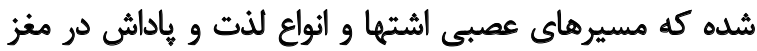

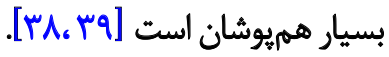
از آنجا كه يُروهش هاي مختلف شباهت هائى بين اعتياد غذايى

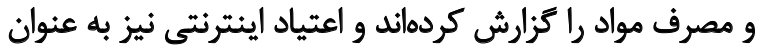

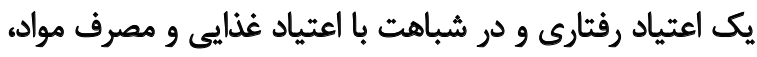

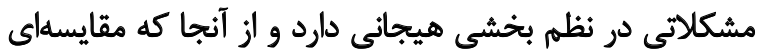

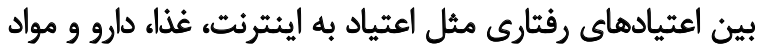

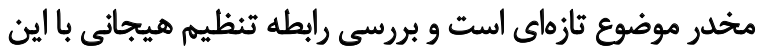

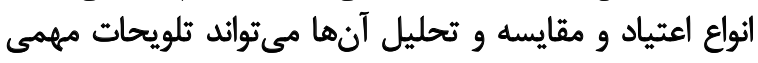

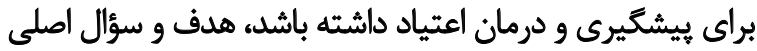

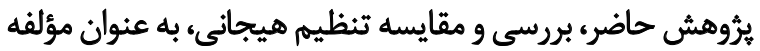

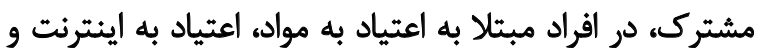
اعتياد به غذا و يك كروه كنترل بهنجار است.

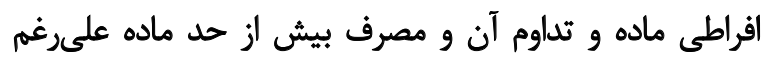

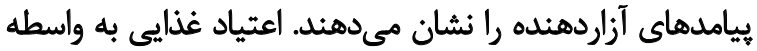

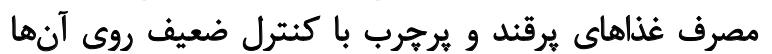

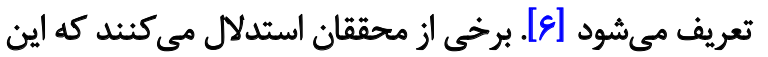

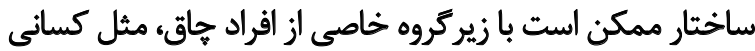

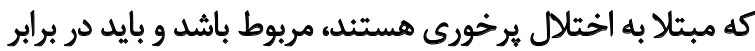

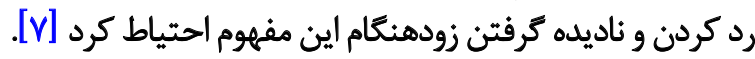
به نظر مىرسد كه هم اعتياد غذايى و هم اختلال برخورى به به

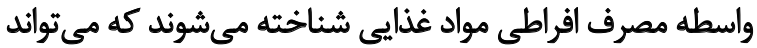

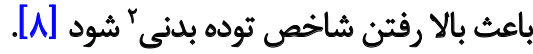

مطالعات اخير كزارش كرداند كه هV درصد از بيماران مبتلا

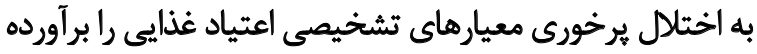

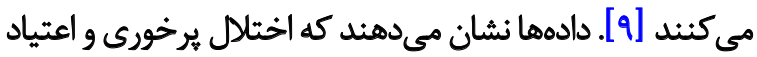

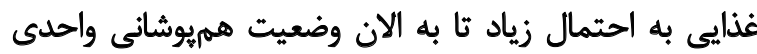

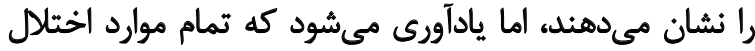

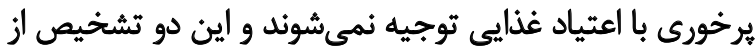

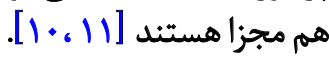
اعتياد غذايى، مثل وابستخى به مواد، با عاطفه منفى، اختلالات

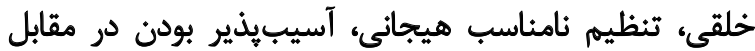

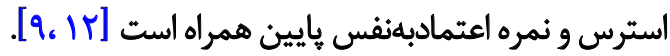
اين يافتهها نشان مى دهند كه مشابه افرادى كه مبتلا به

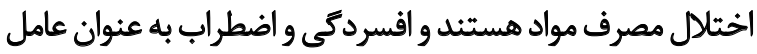

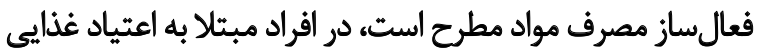

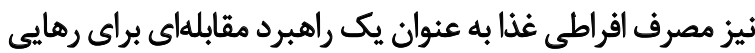

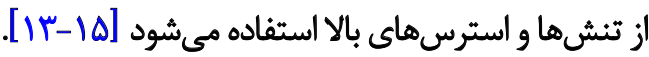

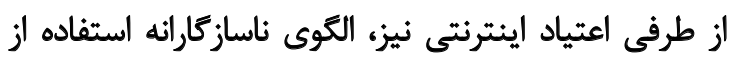

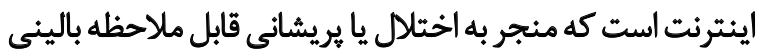

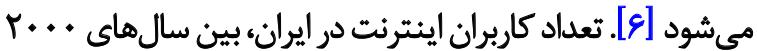

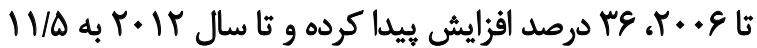

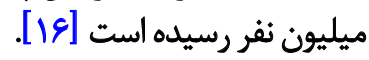
تاكنون مطالعات شيوشناسى اعتياد غذايى در ايران انجام

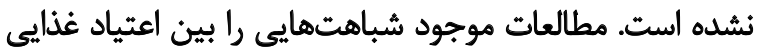

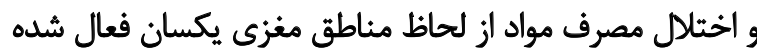
يكان [IV، IA]

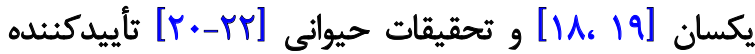

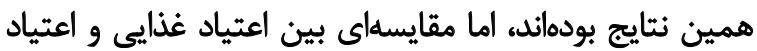
اينترنتى صورت نكرفته است. به طور كلى، دركير شدن در رفتارهاى ناسازگارانهاى

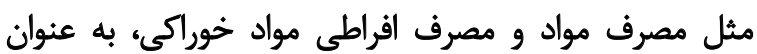


فلينت، كيرهارت، كاربين و براونل [Fr] مقياس اعتياد غذايى

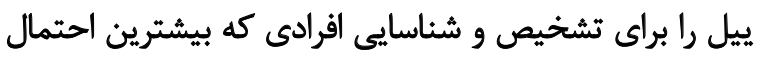

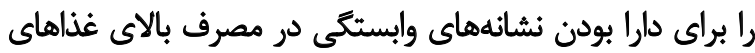

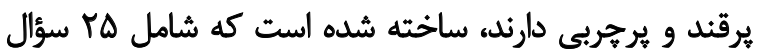

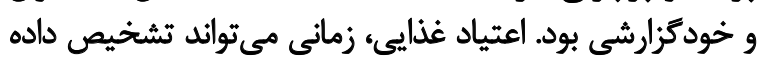

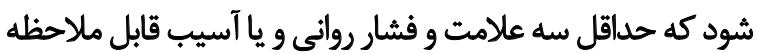
بالينى در فرد موجود باشد.

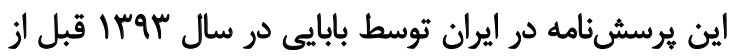

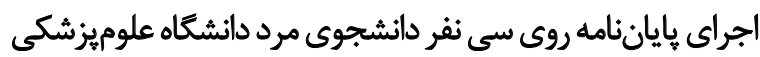

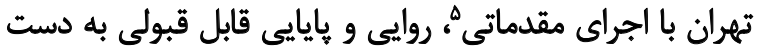

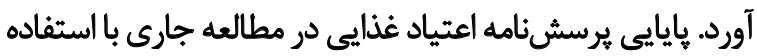

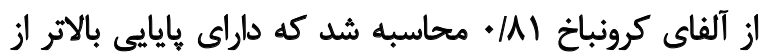

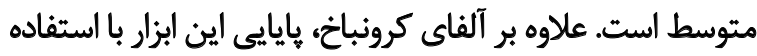

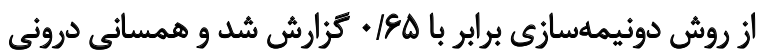

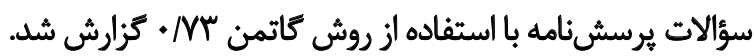

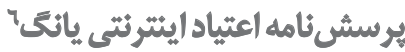

يرسش نامه استانداردشده اعتياد اينترنتى ابتدا توسط كيمبرلى إنى إنها

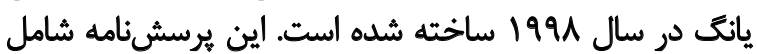

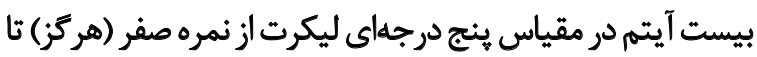

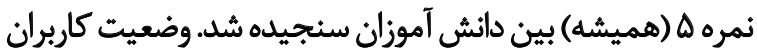

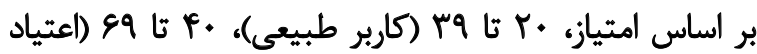

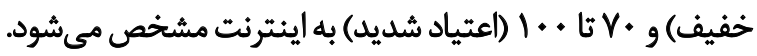

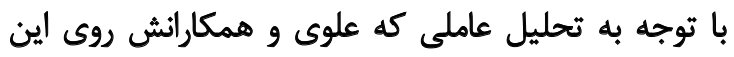

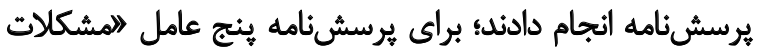

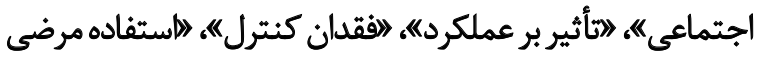

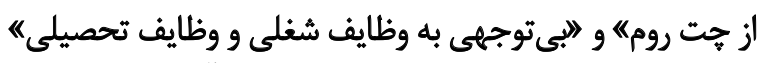

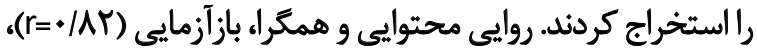

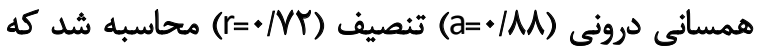

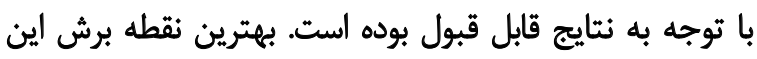

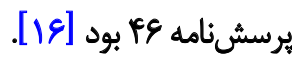

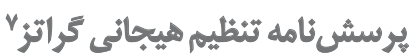

اين يرسشنامه توسط كراتز ساخته شده است. اين مقياس

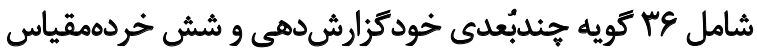

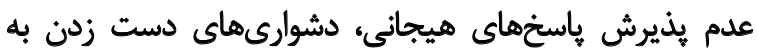

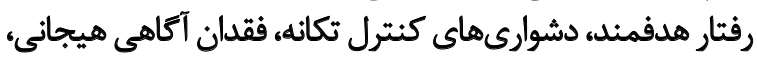

4. Yale Food Addiction Test (YFAS)

5. Pilot

6. Internet Addiction Test (IAT)

7. Difficulties in Emotion Regulation Scale (DERS)
طرح كلى مطالعه در هارجوب يك مطالعه علتّ مقايسهاي بود.

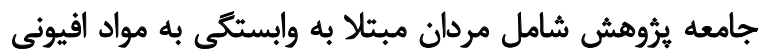

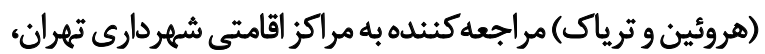

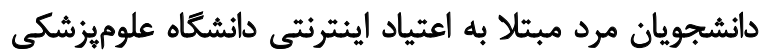

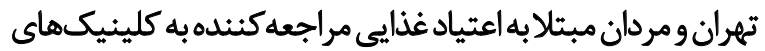
درمان جاقى بيمارستان امام خمينى تهران بودان

افراد نمونه هر كروه به واسطه مصاحبه تشخيصى توسط

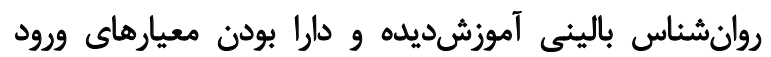

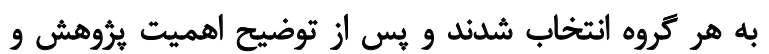

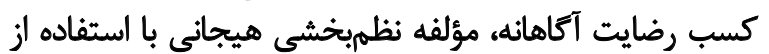

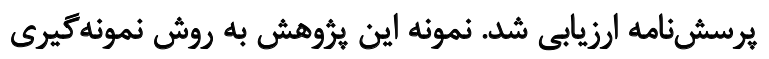

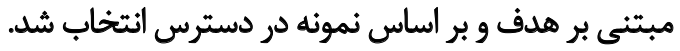
افراد نمونه در هر كروه شامل سى نفر و فقط از جنس مرد بودند

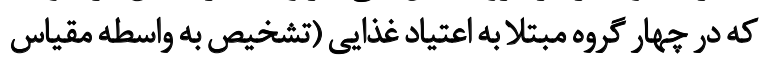

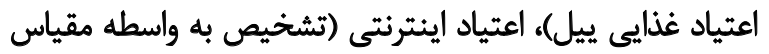

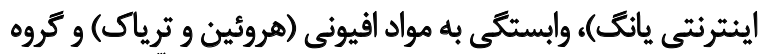

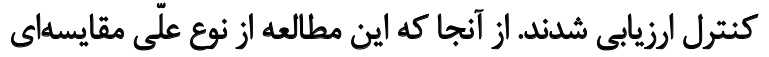

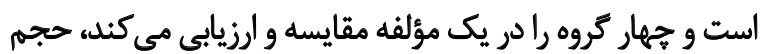

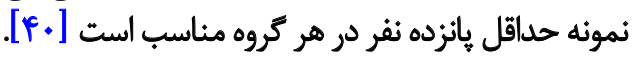

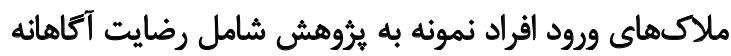

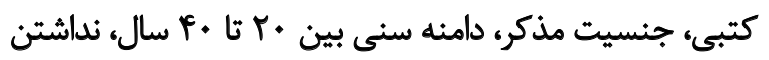

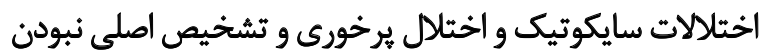

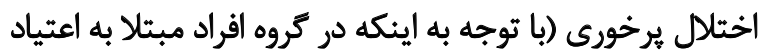

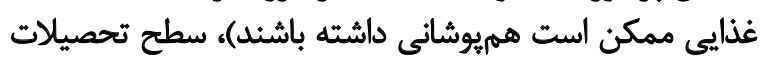

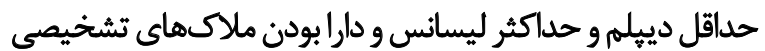

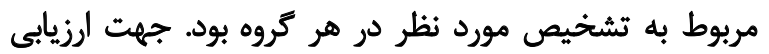
ملاكهاى ورود از ابزارهاى زير استفاده شد:

\section{هصاحبه باليني نيمه ساختّار ياقثته براي انمتلالات محور يك"}

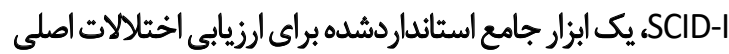

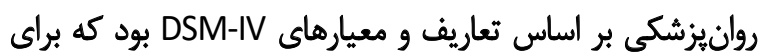

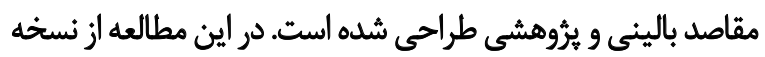

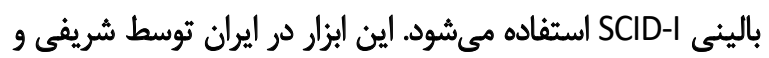

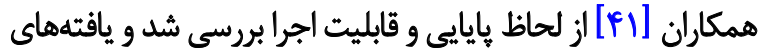

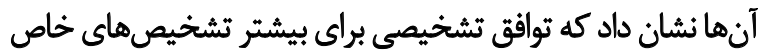
و كلى، متوسط تا خوب (كاياى بالاتر از عان •) بود. 
دارد. براي بررسى اينكه بين كدام كروهها از نظر تنظيم هيجان

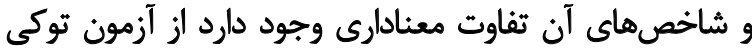

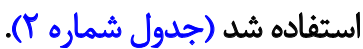

به لحاظ شاخص كلى دشوارى در نظمهبخشى هيجاني و تمام

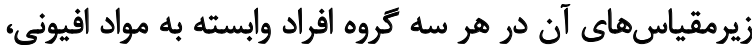

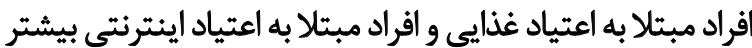

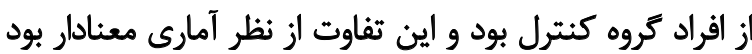

. $(P<\cdot / \cdot \Delta)$

شاخص كلى دشوارى در نظمبخشى هيجانى، در افراد وابسته

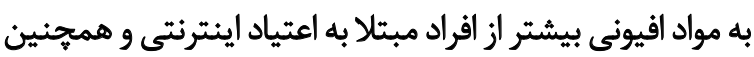

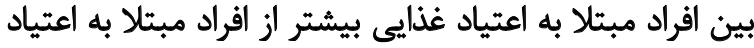

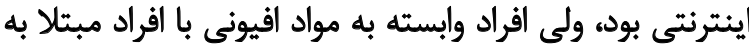
اعتياد غذايى در اين شاخص تفاوت وانت معنادارى نداشتند.

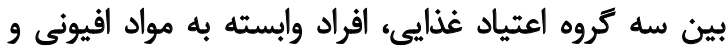

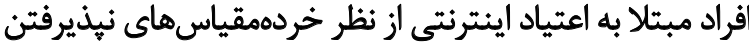

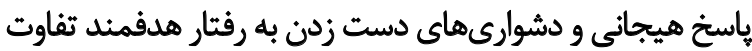

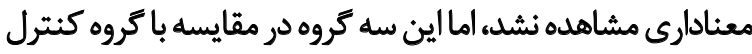

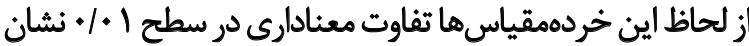

دادند.

در شاخص دشوارى كنترل تكانه همجنين تفاوت معنادارى

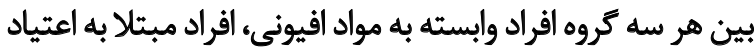

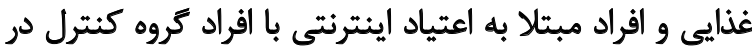

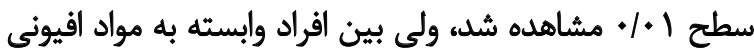

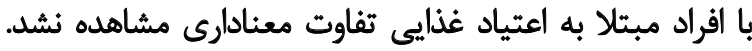

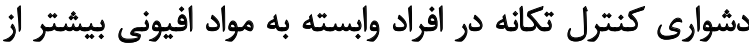

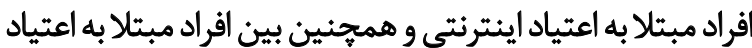

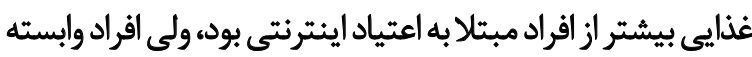

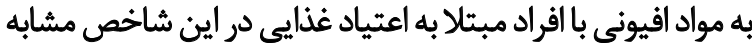

بوند.

در شاخص فقدان آكاهى هيجاني، در هر سه كروه افراد وابسته

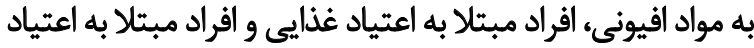

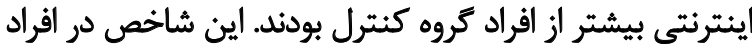

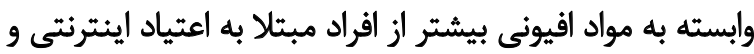

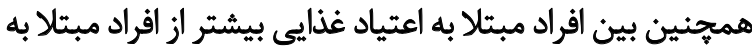

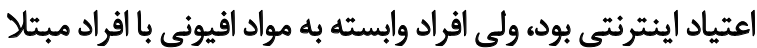

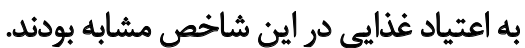

دسترسى محدود به راهبردها در هر سه گروه افراد وابسته به

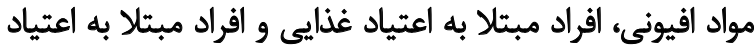

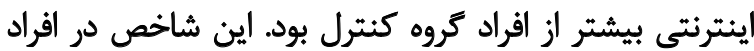

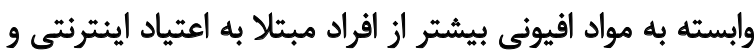

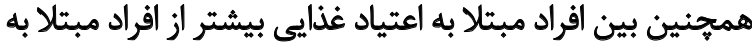

دسترسى محدود به راهبردهاي تنظيم هيجانى و فقدان شفافيت

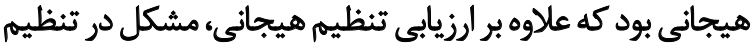

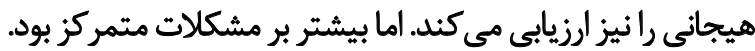

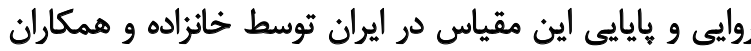

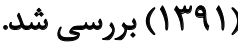
نتايج حاصل از بررسى يايايى اين مقياس نيز نشان داد

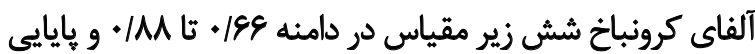

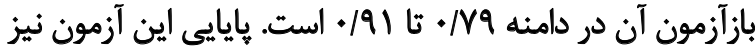

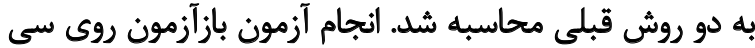

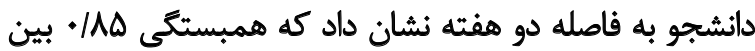

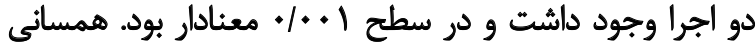

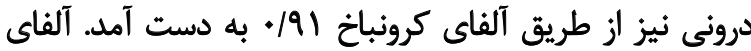

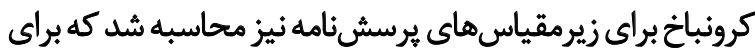

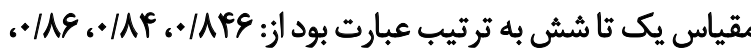

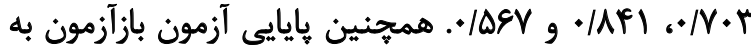

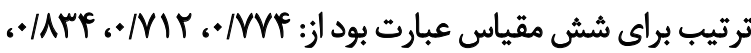

[Fr] •/DYq, •/AFr.•/Fq1

تحليل آمارى

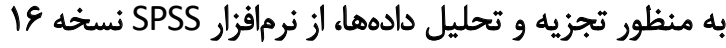

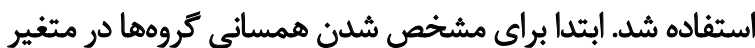

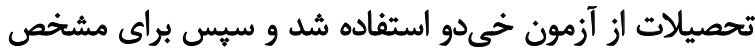

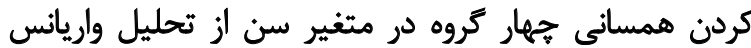

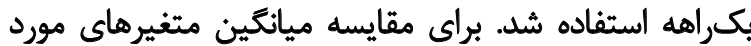

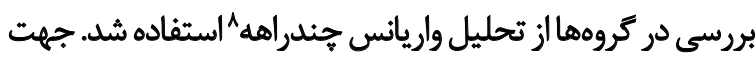

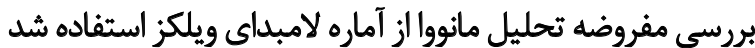

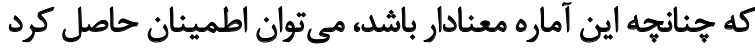
كه امكان استفاده از آزمون مانووا وجود داردا ماندان

نتايج بررسى اثر متغيرهاى گروه يرؤوهى با استفاده از لامبدا

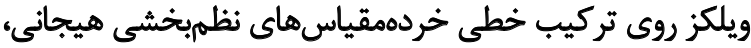

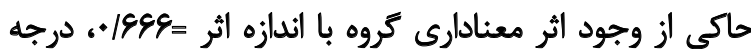

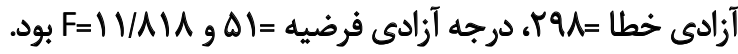

ياقتهها

به طور كلى •ll إنفر در اين مطالعه شركت كردند. تعداد افراد

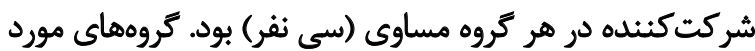

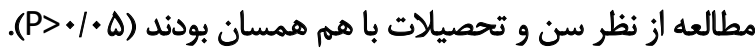

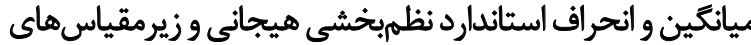

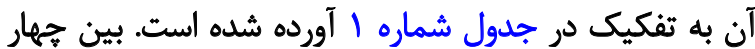

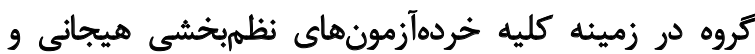

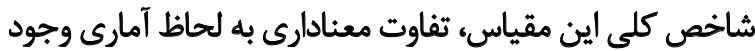

\section{MANOVA}


جدول ا. تحليل واريانس جندمتغيره شاخصهاى دشوارى در نظمبخشى هيجانى

\begin{tabular}{|c|c|c|c|c|c|c|c|}
\hline اندازه اثر & سطح معنادارى & $\mathbf{F}$ & ميانغين مجذورات & مجموع مجذذورات & ميائكين دانحراف استاندارود & نوع كُوها & شُاخص \\
\hline.$/ r \cdot$. & .10 .1 & V/IV. & AD/FAY & $\operatorname{Mer} / \mathrm{Mr}^{\mathrm{s}}$ & $\begin{array}{l}\mid r / N E \pm \varphi / \cdot q \\
\mid \varphi / N \cdot \pm r / V \cdot \\
|r / q \varepsilon \pm r / 4| \\
1 . / r \& \pm r / m \mid\end{array}$ & 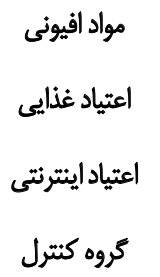 & ثيذيرفتن ياسخهاي هيجاني \\
\hline . MIIP & $.1 . .1$ & $1 r / 1 r q$ & $\mid E q / m$ & EVE/IYA & 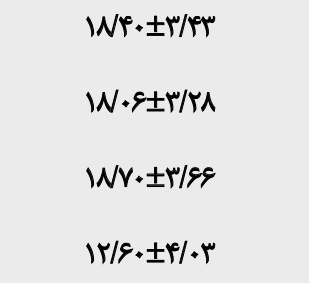 & 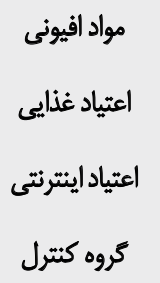 & دشوارى در رفتار هدفمند \\
\hline.$/ \Delta V T$ & .10 .1 & PNFII & DQV/DPF & $M a . /$ lve & $\begin{array}{l}r / r E \pm r / / r \\
\mid Q / q u \pm F / \cdot 1) \\
\mid \Delta / \& r \pm \Delta / \cdot q \\
q / q+ \pm r / / f\end{array}$ & 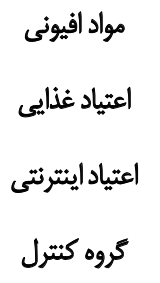 & دشوارى كتترل تكائه \\
\hline . req & .1 .01 & VEAMF & TET/VAY & $1 . \Delta \Delta / I T V$ & $\begin{array}{l}r \cdot / \Delta r \pm \varphi / r r \\
r \cdot / q \cdot \pm \psi / r 1 \\
1 q / q \cdot \pm r / r q \\
\mid r / q r \pm r /+1\end{array}$ & 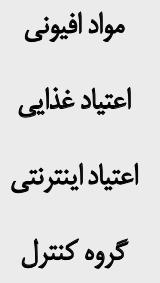 & فقدان أكاهي هيجائى \\
\hline IgT. & $.1 .+1$ & fe/97q & VAV/\&AY & M. MNVA. & 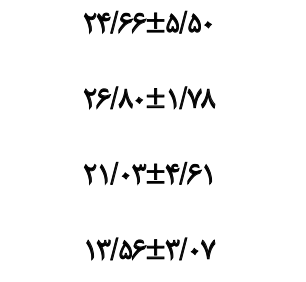 & 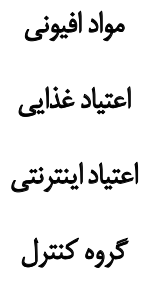 & دسترسى هحدود به راهبردها \\
\hline 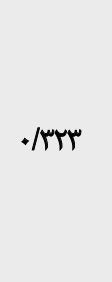 & $.1 . .1$ & IT/MT & $\ln T / \lambda \cdot r$ & $991 / 7+1$ & 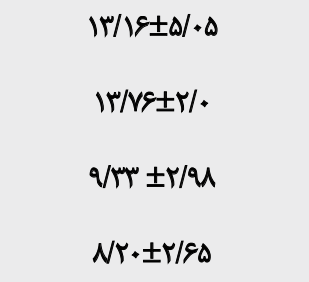 & 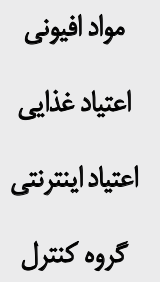 & ايهام هيجانى \\
\hline .1089 & $.1 .+1$ & rV/AqF & $1+F E V / D I Y$ & FIAFEl.9A & 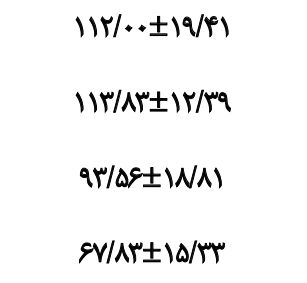 & 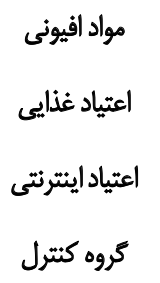 & شاخص كلى \\
\hline
\end{tabular}


جدول r. نتايج آزمون توكى در مقايسه جيندكائه بين كروها در شاخصهاى دشوارى در نظمبخشى هيجانى

\begin{tabular}{|c|c|c|c|c|}
\hline سطح معنادارى & انحراف استاندارد土ثفاوت ميانكين & 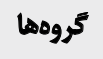 & 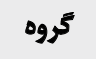 & شاخصها \\
\hline . $/ \mathrm{NA}$ & $-\cdot / A \Psi \pm \cdot / M$ & غذذا & & \multirow{6}{*}{ عدم هذيرش ياسخ هاي هيجائى } \\
\hline.$/ 99$ & $-\cdot / 1 \cdot \pm \cdot / M$ & اينترنت & مواد & \\
\hline$+1+1$ & $r / \Delta+ \pm \cdot / M$ & كتترل & \multirow{3}{*}{ غذا ا } & \\
\hline /AP & $\cdot / N^{H} \pm \cdot / M$ & اينترنت & & \\
\hline $.1 . .1$ & $F / M T \pm \cdot / M$ & كتترل ك & & \\
\hline $.1 .+1$ & $r / s \cdot \pm \cdot / M$ & كتترل & ايثترنت & \\
\hline $.14 \mathrm{u}$ & $. / R \pm . / q \pi$ & غذا & \multirow{5}{*}{ 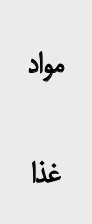 } & \multirow{6}{*}{ دشوارى هاى دست زدن به رفتار هدفمند } \\
\hline / ME & 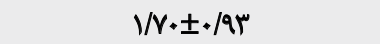 & ايتترنت & & \\
\hline $.1 . .1$ & $\Delta / A \cdot \pm \cdot / 9 T$ & 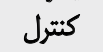 & & \\
\hline.$/ 48$ & V/rED./ar & ايتترنت & & \\
\hline.. .1 & 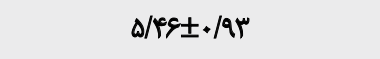 & 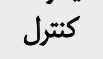 & & \\
\hline.$/ .+1$ & $4 / 1 \cdot \pm \cdot / 9 \pi$ & 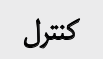 & 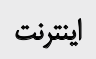 & \\
\hline.$/ 29$ & V/TrtV/.1 & غذا & \multirow{5}{*}{ 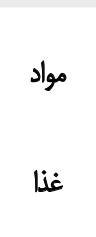 } & \multirow{6}{*}{ تشوارى هاي كتثرل تكانه } \\
\hline $.1 .+1$ & $\Delta / N V^{\prime} \pm V / * 1$ & اينترنت & & \\
\hline$+1+\infty 1$ & $\mid 1 / 4 \& \pm 1 / \cdot 1$ & كنترل & & \\
\hline $.1 .+1$ & $r / \mu \cdot \pm V / \cdot 1$ & ايتترنت & & \\
\hline $.1 . .1$ & $1.1 . r \pm 1 / .1$ & 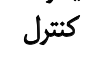 & & \\
\hline.$/+1$ & $\Delta / N^{\prime} \pm 1 /+1$ & كتترل & اينترنت & \\
\hline $1 / \cdot$ & $-.1 .9 \pm 1 / .4$ & غذا & \multirow{3}{*}{ 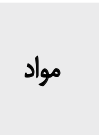 } & \multirow{6}{*}{ فقدان آكاهى هيجانى } \\
\hline.. .4 & $r / g r \pm 1 / r$ & ايتترنت & & \\
\hline $.1 .+1$ & $8 / 9 . \pm 1 / . r$ & كنترل & & \\
\hline$\%$ & $r / v \cdot \pm V / r$ & اينترنت & \multirow{2}{*}{ 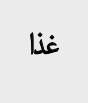 } & \\
\hline$+1+\infty 1$ & $\varepsilon / q \varepsilon \pm 1 / \circ r$ & كنترل & & \\
\hline .101 & $r / K \varepsilon \pm V / r$ & كتترل & اينترئت & \\
\hline.$/ N$ & $-r / / r \pm V / \cdot r$ & غذا & \multirow{3}{*}{ 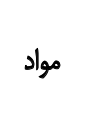 } & \multirow{6}{*}{ دسترسى محلود به راهبردهاى تنظيم هيجانى } \\
\hline...+5 & $r / q r \pm V / r r$ & إيترنت & & \\
\hline $.1 . .1$ & $W / 1 \cdot \pm 1 / \cdot r$ & كتثرل & & \\
\hline $.1 . .1$ & $\Delta / V E \pm V / . r$ & ايتترنت & \multirow{2}{*}{ غذا } & \\
\hline .1 .1 & $\mid r / r m \pm 1 / r$ & كنترل & & \\
\hline $.1 . .1$ & $V / F A \pm V / r$ & 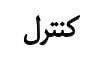 & 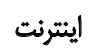 & \\
\hline.$/ 91$ &.$-|9 . \pm \cdot / 9|$ & غذا & \multirow{5}{*}{ غذا } & \multirow{6}{*}{ فقدان شفافيت هيجانى } \\
\hline.$/ . .1$ & $r / A r \pm \cdot / 91$ & ايتترنت & & \\
\hline $.1 . .1$ & $P / Q \varepsilon \pm \cdot / 91$ & 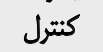 & & \\
\hline $.1+.1$ & $r / H \Psi \pm \cdot / 91$ & ايتترنت & & \\
\hline $.1 .+1$ & $\Delta / \Delta \& \pm \cdot / q)$ & كثترل & & \\
\hline.$/ .1$ & $\Delta /|r \pm \cdot / q|$ & 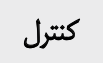 & 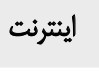 & \\
\hline .194 & - $/ / A T \pm F / M T$ & غذا غذا & \multirow{3}{*}{ 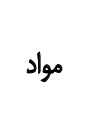 } & \multirow{6}{*}{ شاخص كلى دشوارى در نظهيخشى هيجانى } \\
\hline $.1 .+1$ & WPTETRYY & اينترنت & & \\
\hline$+1+\infty 1$ & $F \Psi / N E \pm T / M T$ & كنترل & & \\
\hline$+1+.1$ & $r \cdot / r E \pm F / K r$ & ايتترنت & \multirow{2}{*}{ 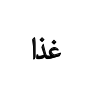 } & \\
\hline $.1 . .1$ & $P Q / . . \pm F / M T$ & كتثرل & & \\
\hline $.1+.1$ & $r \Delta / M^{\mu} \pm T / M T$ & 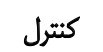 & اينترنت & \\
\hline
\end{tabular}




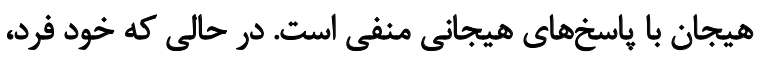

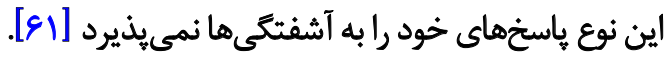

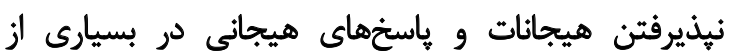

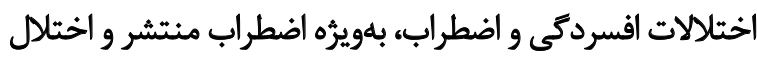

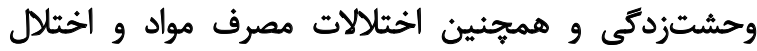

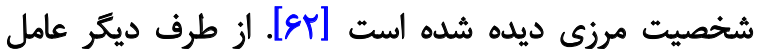

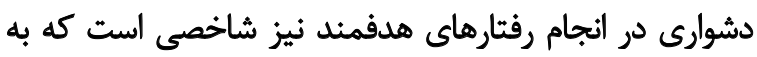

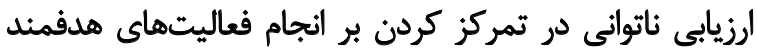

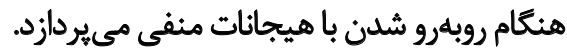

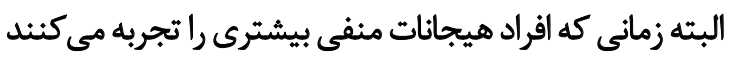

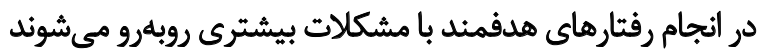

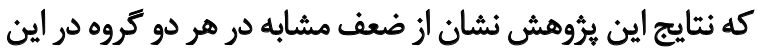

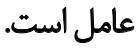

تأثير هيجائات منفى بر انجام اين رفتارها، به خزانه راهبردهاي

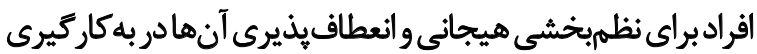

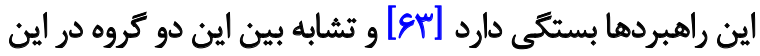

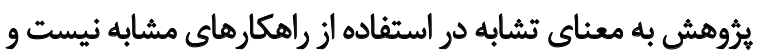
اين امر خود نياز به بررسى جدابه دانه دارد.

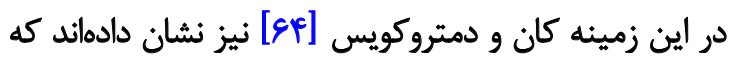

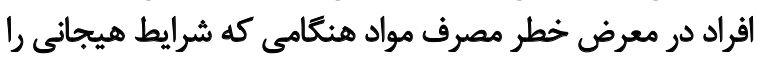

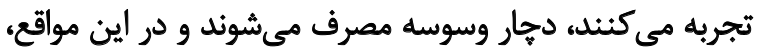

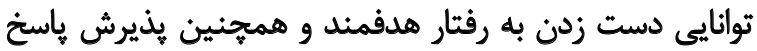
هيجانى ضربه تير مصرف مواد است.

در ساير شاخصهاى نظمبخشى هيجانى هم افراد كروه وابسته

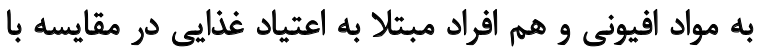

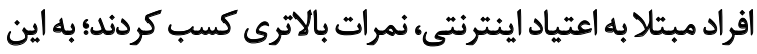

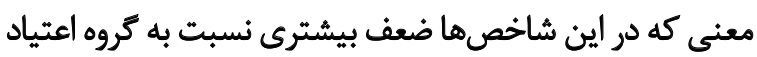

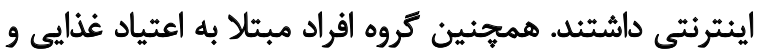

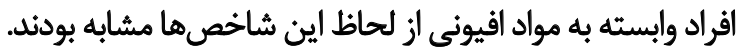

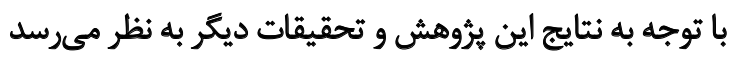

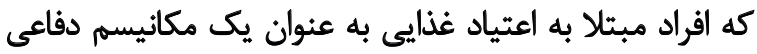

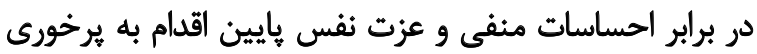

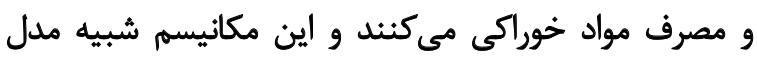

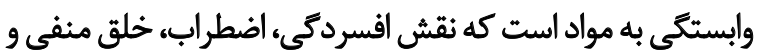

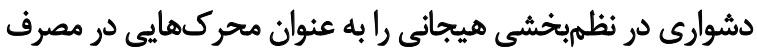

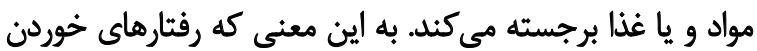

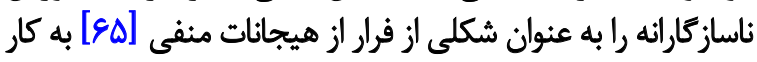

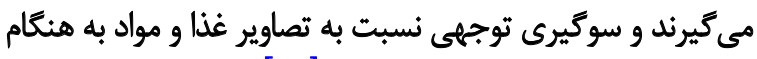

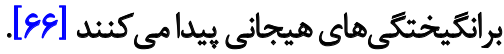

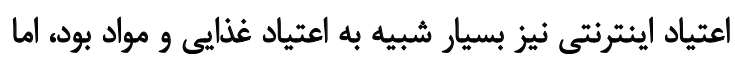

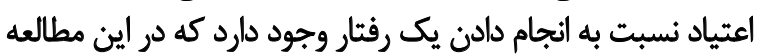

اعتياد اينترنتى بود، ولى اقراد وابسته به مواد افيونى با اقراد مبتلا به إنه

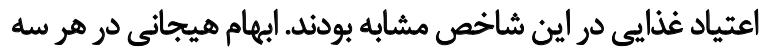

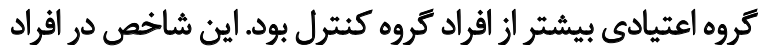

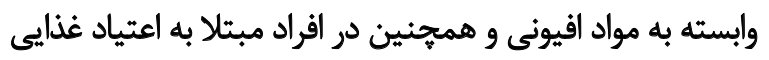

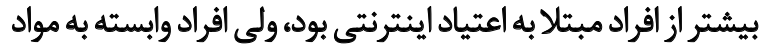

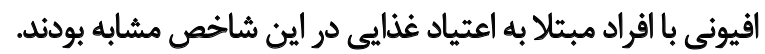

بحث

نتيجه يروهش حاضر نشان داد كه در كروههاي افراد وابسته

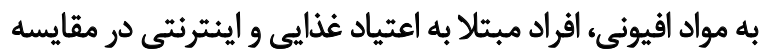

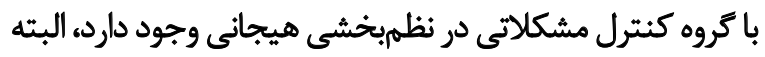

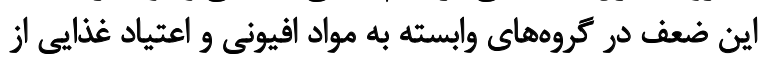

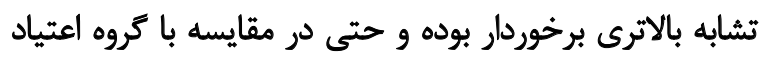
اينترنتى ضعف بيشترى از اين لحاظ نشان دادند.

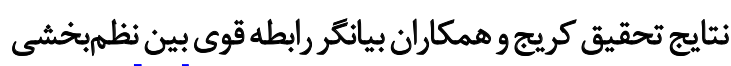

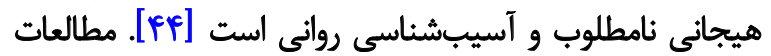

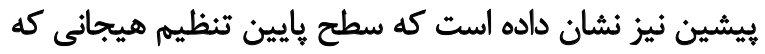

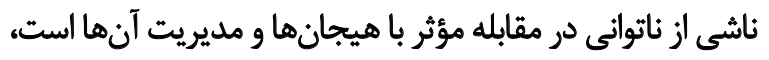

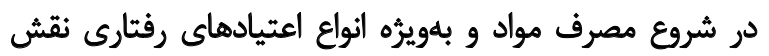
اساسى دارد [Fa-FV]

همانطور كه تحقيقات كستردهاي در زمينه وابستكى به مواد مواد

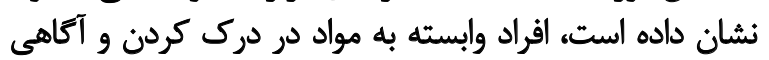

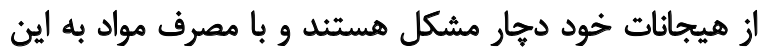

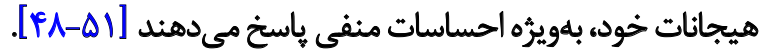

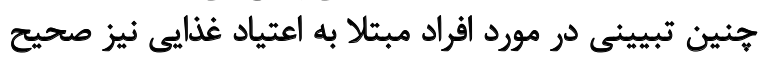

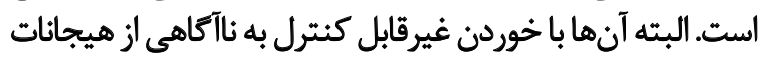

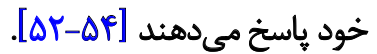

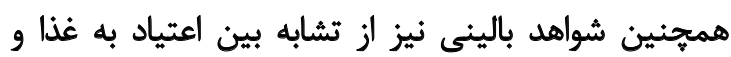

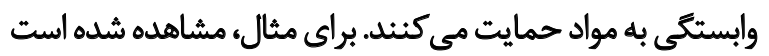

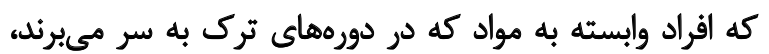

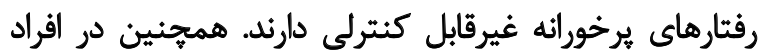

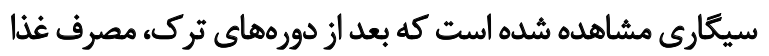

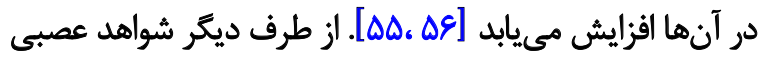

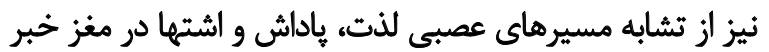

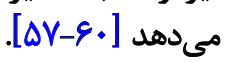

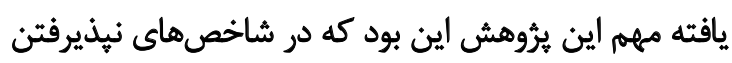

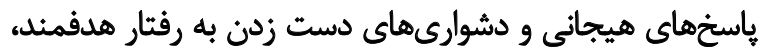

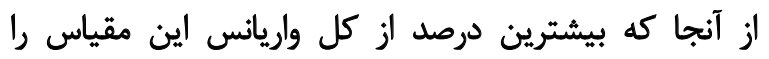

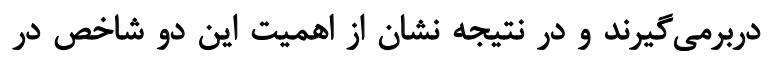

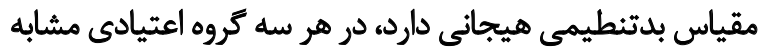

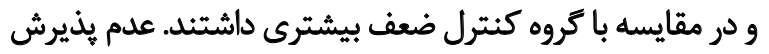

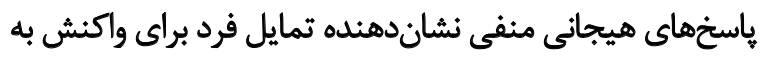




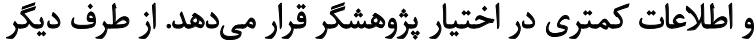

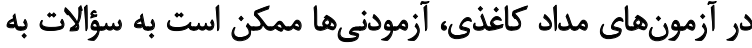

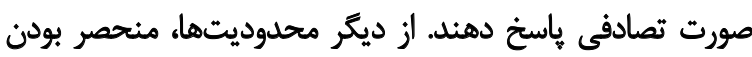

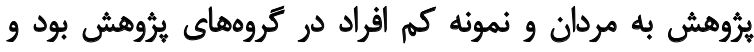

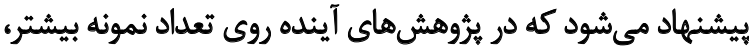

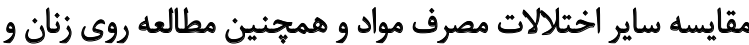

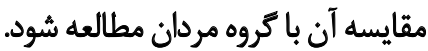

ملاحظات اخلاقثى

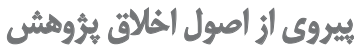

در اين مطالعه رضايت آكاهانه كتبى از مشاركت كنيندكان جهت

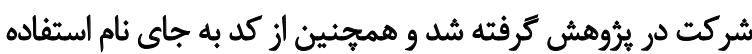

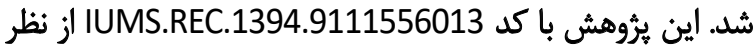
كميته اخلاق در بروهش دانشكاه تأييد شد.

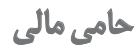

اين مقاله بركرفته از يايانتامه كارشناسىارشد آقاى شيرزاد بابايى

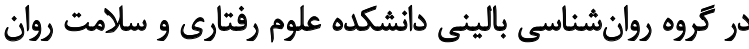

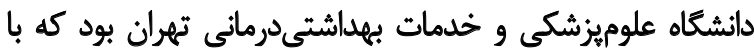

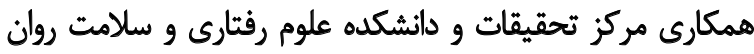

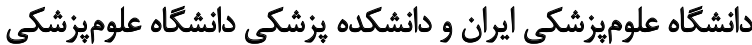

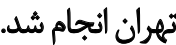

$$
\text { مشاركت نويسندكان }
$$

مفهومسازى، روششناسى، نكارش ييشنويس، ويراستارى و

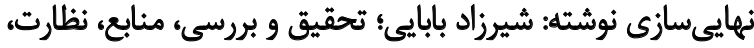

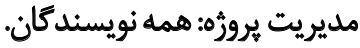

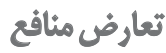

بنابر اظهار نويسندكان هيجّونه تعارض منافعى در اين مطالعه و

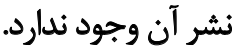

ضعف بيشتر نظمهبخشى هيجانى در دو اعتياد ديكر نسبت به اينترنت

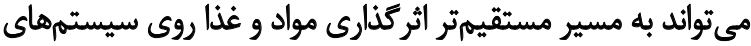

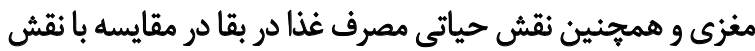

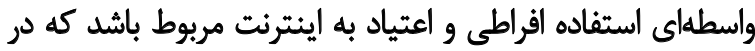
تحقيقات مختلف ويرگكى هاى مشابه اين سه نوع اعتياد باعث شده كه

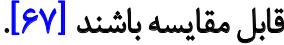

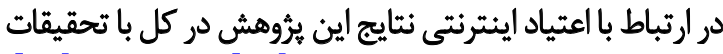

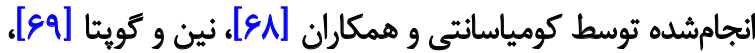

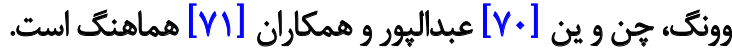

البته در اين يُروهش ابهام هيجاني يا نبود وضوح هيجانى داراي

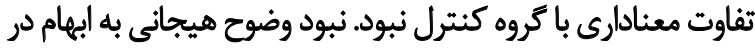

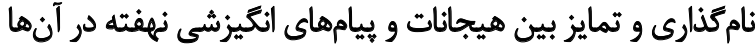

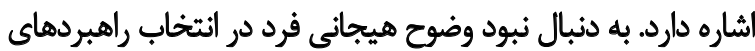

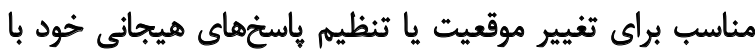
مشكلات بسيارى روبهرو مىشود.

ابهام در هيجانات از ويثگى هاى اصلى بسيارى از اختلالات، از

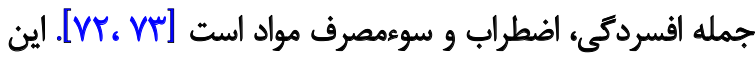

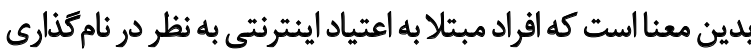

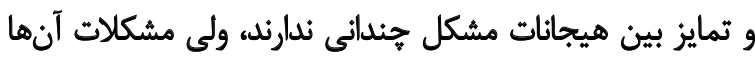

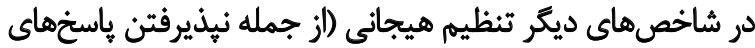

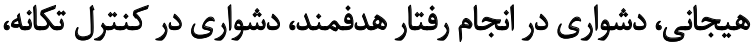

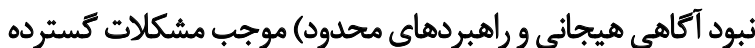

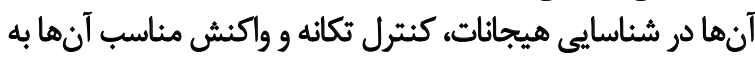

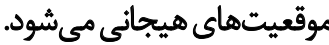

$$
\text { تثيجهئرى }
$$

در كل نتايج اين يُروهش با نتايج نظريه خوددرمانى خانتزيان

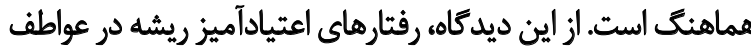

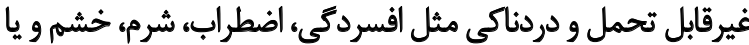

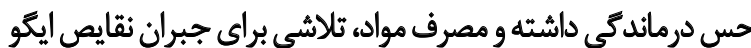

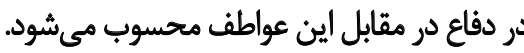

اين ديدكاه با مشاهدات بالينى و مطالعات فراوائى كه بر نقش

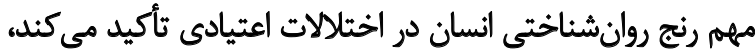

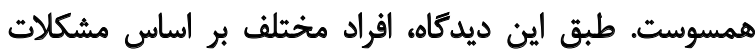

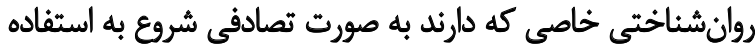

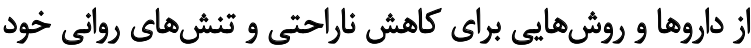

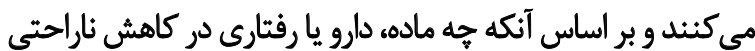

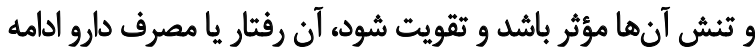

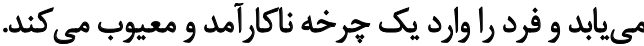

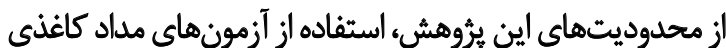
بود كه اين نوع آزمونها در مقايسه با مصاحبه تخصصى اعتبار كمتر 


\section{References}

[1] Starcevic V. Behavioural addictions: Achallengefor psychopathology and psychiatric nosology. Australian \& New Zealand Journal of Psychiatry. 2016; 50(8):721-5. [DOI:10.1177/0004867416654009] [PMID]

[2] Joranby L, Pineda KF, Gold MS. Addiction to food and brain reward systems. Sexual Addiction \& Compulsivity. 2005; 12(23):201-17. [DOI:10.1080/10720160500203765]

[3] Merlo LJ, Klingman C, Malasanos TH, Silverstein JH. Exploration of food addiction in pediatric patients. Journal of Addiction Medicine. 2009; 3(1):26-32. [DOI:10.1097/ADM.0b013e31819638b0] [PMID] [PMCID]

[4] Sadock BJ, Sadock VA. Kaplan and Sadock's synopsis of psychiatry: Behavioral sciences/clinical psychiatry. New York: Lippincott Williams \&Wilkins; 2011. https:/ / books.google.com/boo $\mathrm{ks}$ ?hl=fa\&lr=\&id=fFi7DR2hmaIC\&oi=fnd\&pg=PA7\&dq=

[5] Amin-Esmaeili M, Rahimi-Movaghar A, Sharifi V, Hajebi A, Radgoodarzi R, Mojtabai R, et al. Epidemiology of illicit drug use disorders in Iran: prevalence, correlates, comorbidity and service utilization results from the Iranian mental health survey. Addiction. 2017; 111(10):1836-47. [PMID] [DOI:10.1111/add.13453]

[6] Gearhardt AN, Roberto CA, Seamans MJ, Corbin WR, Brownell KD. Preliminary validation of the Yale Food Addiction Scale for children. Eating Behaviors. 2013; B14(4):508-12. [DOI:10.1016/j. eatbeh.2013.07.002] [PMID] [PMCID]

[7] Avena NM, Gearhardt AN, Gold MS, Wang GJ, Potenza MN. Tossing the baby out with the bathwater after a brief rinse? The potential downside of dismissing food addiction based on limited data. Nature Reviews Neuroscience. 2012; 13(7):514. [DOI:10.1038/nrn3212-c1] [PMID]

[8] Gearhardt A, White MA, Potenza MN. Binge eating disorder and food addiction. Current Drug Abuse Reviews. 2011; 4(3):201-7. [D OI:10.2174/1874473711104030201] [PMID]

[9] Gearhardt AN, White MA, Masheb RM, Morgan PT, Crosby $\mathrm{RD}$, Grilo CM. An examination of the food addiction construct in obese patients with binge eating disorder. International Journal of Eating Disorders. 2012; 45(5):657-63. [DOI:10.1002/eat.20957] [PMID] [PMCID]

[10] Avena N, Bocarsly M E, Hoebel B G, Gold M S. Overlaps in the nosology of substance abuse and overeating: The translational implications of "food addiction". Current Drug Abuse Reviews. 2011; 4(3):133-9. [DOI:10.2174/1874473711104030133] [PMID]

[11] Marcus MD, Wildes JE. Obesity: Is it a mental disorder? International Journal of Eating Disorders. 2009; 42(8):739-53. [DOI:10.1002/eat.20725] [PMID]

[12] Davis C, Curtis C, Levitan RD, Carter JC, Kaplan AS, Kennedy JL. Evidence that 'food addiction' is a valid phenotype of obesity. Appetite. 2011; 57(3):711-7. [DOI:10.1016/j.appet.2011.08.017] [PMID]

[13] Holahan CJ, Moos RH, Holahan CK, Cronkite RC, Randall PK. Drinking to cope, emotional distress and alcohol use and abuse: a ten-year model. Journal of Studies on Alcohol. 2001; 62(2):190-8. [DOI:10.15288/jsa.2001.62.190] [PMID]

[14] Nunes EV, Rounsaville BJ. Comorbidity of substance use with depression and other mental disorders: From Diagnostic and
Statistical Manual of Mental Disorders, (DSM-IV) to DSM-V. Addiction. 2006; 101:89-96. [DOI:10.1111/j.1360-0443.2006.01585.x] [PMID]

[15] Thorberg FA, Lyvers M. Negative Mood Regulation (NMR) expectancies, mood, and affect intensity among clients in substance disorder treatment facilities. Addictive Behaviors. 2006; 31(5):81120. [DOI:10.1016/j.addbeh.2005.06.008] [PMID]

[16] Alavi SS, Maracy MR, Jannatifard F, Eslami M. The effect of psychiatric symptoms on the internet addiction disorder in Isfahan's University students. Journal of Research in Medical Sciences 2011; 16(6):793-800. [PMCID] [PMID]

[17] Schulte EM, Grilo CM, Gearhardt AN. Shared and unique mechanisms underlying binge eating disorder and addictive disorders. Clinical Psychology Review. 2016; 44:125-39. [DOI:10.1016/j.cpr.2016.02.001] [PMID] [PMCID]

[18] Volkow ND, Koob GF, McLellan AT. Neurobiologic advances from the brain disease model of addiction. New England Journal of Medicine. 2016; 374(4):363-71. [DOI:10.1056/NEJMra1511480] [PMID] [PMCID]

[19] Majuri J, Joutsa J, Johansson J, Voon V, Alakurtti K, Parkkola R, et al. Dopamine and opioid neurotransmission in behavioral addictions: a comparative PET study in pathological gambling and binge eating. Neuropsychopharmacology. 2017; 42(5):1169-77. [DOI:10.1038/npp.2016.265] [PMID] [PMCID]

[20] Becker JB, Koob GF. Sex differences in animal models: Focus on addiction. Pharmacological Reviews. 2016; 68(2):242-63. [DOI:10.1124/pr.115.011163] [PMID] [PMCID]

[21] Hone-Blanchet A, Fecteau S. Overlap of food addiction and substance use disorders definitions: Analysis of animal and human studies. Neuropharmacology. 2014; 85:81-90. [DOI:10.1016/j. neuropharm.2014.05.019] [PMID]

[22] Robbins T, Clark L. Behavioral addictions. Current Opinion in Neurobiology. 2015; 30:66-72. [DOI:10.1016/j.conb.2014.09.005] [PMID]

[23] Berenson AB, Laz TH, Pohlmeier AM, Rahman M, Cunningham KA. Prevalence of food addiction among low-income reproductive-aged women. Journal of Women's Health. 2015; 24(9):740-4. [DOI:10.1089/jwh.2014.5182] [PMID] [PMCID]

[24] Chou WP, Yen CF, Liu TL. Predicting effects of psychological inflexibility/experiential avoidance and stress coping strategies for internet addiction, significant depression, and suicidality in college students: A prospective study. International Journal of Environmental Research and Public Health. 2018; 15(4):788. [DOI:10.3390/ijerph15040788] [PMID] [PMCID]

[25] Mitchell KS, Wolf EJ. PTSD, food addiction, and disordered eating in a sample of primarily older veterans: The mediating role of emotion regulation. Psychiatry Research. 2016; 243:23-9. [DOI:10.1016/j.psychres.2016.06.013] [PMID] [PMCID]

[26] Burnay J, Billieux J, Blairy S, Larøi F. Which psychological factors influence Internet addiction? Evidence through an integrative model. Computers in Human Behavior. 2015; 43:28-34. [DOI:10.1016/j.chb.2014.10.039]

[27] Pivarunas B, Conner BT. Impulsivity and emotion dysregulation as predictors of food addiction. Eating Behaviors. 2015; 19:9-14. [DOI:10.1016/j.eatbeh.2015.06.007] [PMID]

[28] Farstad S. Examining the short-term longitudinal relationships between emotion regulation and addictive behaviors among 
community women. [Unpublished PhD. dissertation)]. Calgary: University of Calgary; 2017. [DOI:http://dx.doi.org/10.11575/ PRISM/26031]

[29] Verdejo-García A, Bechara A, Recknor EC, Pérez-García M. Negative emotion-driven impulsivity predicts substance dependence problems. Drug \& Alcohol Dependence. 2007; 91(2):213-9. [DOI:10.1016/j.drugalcdep.2007.05.025] [PMID]

[30] Whiteside U, Chen E, Neighbors C, Hunter D, Lo T, Larimer M. Difficulties regulating emotions: Do binge eaters have fewer strategies to modulate and tolerate negative affect? Eating Behaviors. 2007; 8(2):162-9. [DOI:10.1016/j.eatbeh.2006.04.001] [PMID]

[31] Amiri S, Naseri Tamrin K. [The role of cognitive emotion regulation strategies, impulsivity and extraversion in the tendency of the internet addiction in the students of Urmia university, in 2014 (Persian)]. Pajouhan Scientific Journal. 2015; 14(1):1-11. https:// www.sid.ir/en/journal/ViewPaper.aspx?id=518999

[32] Eichen DM, Chen EY, Schmitz MF, Arlt J, McCloskey MS. Addiction vulnerability and binge eating in women: Exploring reward sensitivity, affect regulation, impulsivity \& weight/shape concerns. Personality and Individual Differences. 2016; 100:16-22. [DOI:10.1016/j.paid.2016.03.084] [PMID] [PMCID]

[33] Griffin KW, Lowe SR, Acevedo BP, Botvin GJ. Affective selfregulation trajectories during secondary school predict substance use among urban minority young adults. Journal of Child \& Adolescent Substance Abuse. 2015; 24(4):228-34. [DOI:10.1080/106782 8X.2013.812530] [PMID] [PMCID]

[34] Weiss NH, Sullivan TP, Tull MT. Explicating the role of emotion dysregulation in risky behaviors: A review and synthesis of the literature with directions for future research and clinical practice. Current opinion in Psychology. 2015; 3:22-9. [DOI:10.1016/j. copsyc.2015.01.013] [PMID] [PMCID]

[35] Parker JD, Taylor RN, Eastabrook JM, Schell SL, Wood LM. Problem gambling in adolescence: Relationships with internet misuse, gaming abuse and emotional intelligence. Personality and Individual Differences. 2008; 45(2):174-80. [DOI:10.1016/j. paid.2008.03.018]

[36] Spitzer RL, Yanovski S, Wadden T, Wing R, Marcus MD, Stunkard A, et al. Binge eating disorder: its further validation in a multisite study. international Journal of Eating Disorders. 1993; 13(2):137-53. [DOI:10.1002/1098-108X(199303)13:23.0.CO;2-R]

[37] Ross HE, Ivis F. Binge eating and substance use among male and female adolescents. International Journal of Eating Disorders. 1999; 26(3):245-60. [DOI:10.1002/(SICI)1098108X(199911)26:33.0.CO;2-R]

[38] Johnson PM, Kenny PJ. Dopamine D2 receptors in addictionlike reward dysfunction and compulsive eating in obese rats. Nature Neuroscience. 2010; 13(5):635. [DOI:10.1038/nn.2519] [PMID] [PMCID]

[39] Volkow ND, Wise RA, Baler R. The dopamine motive system: Implications for drug and food addiction. Nature Reviews Neuroscience. 2017; 18(12):741. [DOI:10.1038/nrn.2017.130] [PMID]

[40] VanVoorhis CW, Morgan BL. Understanding power and rules of thumb for determining sample sizes. Tutorials in Quantitative Methods for Psychology. 2007; 3(2):43-50. [DOI:10.20982/ tqmp.03.2.p043]

[41] Sharifi V, Asadi SM, Mohammadi MR, Amini H, Kaviani H, Semnani $Y$, et al. Reliability and feasibility of the Persian version of the structured diagnostic interview for DSM-IV (SCID). Ad- vances in Cognitive Science. 2004; 6(1):10-22. http:/ / icssjournal. ir/article-1-26-en.html

[42] Flint AJ, Gearhardt AN, Corbin WR, Brownell KD, Field AE, Rimm EB. Food-addiction scale measurement in 2 cohorts of middle-aged and older women-. The American Journal of Clinical Nutrition. 2014; 99(3):578-86. [DOI:10.3945/ajcn.113.068965] [PMID] [PMCID]

[43] Khanzadeh M, Saeediyan M, Hosseinchari M, Edrissi F. [Factor structure and psychometric properties of difficulties in Emotional Regulation Scale (Persian)]. International Journal of Behavioral Sciences. 2012; 6(1):87-96. http:// www.behavsci.ir/article_67768. $\mathrm{html}$

[44] Kraaij V, van EA, Garnefski N, Schroevers MJ, Lo-Fo-Wong D, van EP. Effects of a cognitive behavioral self-help program and a computerized structured writing intervention on depressed mood for HIV-infected people: A pilot randomized controlled trial. Journal of Pastoral Care \& Counseling. 2010; 80(2):200-4 [DOI:10.1016/j.pec.2009.08.014] [PMID]

[45] Hollett KB, Harris N. Dimensions of emotion dysregulation associated with problem video gaming. Addiction Research \& Theory. 2019; 1-8. [DOI:10.1080/16066359.2019.1579801]

[46] Garland EL, Bell S, Atchley R, Froeliger B. Emotion dysregulation in addiction. Oxford: The Oxford Handbook of Emotion Dysregulation; 2018. [DOI:10.1093/oxfordhb/9780190689285.013.23]

[47] Mo PK, Chan VW, Chan SW, Lau JT. The role of social suppor on emotion dysregulation and Internet addiction among Chinese adolescents: A structural equation model. Addictive Behaviors. 2018; 82:86-93. [DOI:10.1016/j.addbeh.2018.01.027] [PMID]

[48] Estevez A, Jauregui P, Sanchez-Marcos I, Lopez-Gonzalez H Griffiths MD. Attachment and emotion regulation in substance addictions and behavioral addictions. Journal of Behavioral Addictions. 2017; 6(4):534-44. [DOI:10.1556/2006.6.2017.086] [PMID] [PMCID]

[49] Hoseiny H, Jadidi M, Nataj LH, Saberi-Zafarghandi MB. [The effect of methadone-maintenance therapy with and without interactive treatment on improving emotion-regulation strategies and resilience among opiate-dependent clients (Persian)]. International Journal of High Risk Behaviors \& Addiction. 2015(1):e23526 [DOI:10.5812/ijhrba.23526] [PMID] [PMCID]

[50] Tull MT, Weiss NH, Adams CE, Gratz KL. The contribution of emotion regulation difficulties to risky sexual behavior within a sample of patients in residential substance abuse treatment. Addictive Behaviors. 2012; 37(10):1084-92. [DOI:10.1016/j.addbeh.2012.05.001] [PMID] [PMCID]

[51] Contreras-Rodríguez O, Albein-Urios N, Martinez-Gonzalez JM, Menchón JM, Soriano-Mas C, Verdejo-García A. The neural interface between negative emotion regulation and motivation for change in cocaine dependent individuals under treatment. Drug and Alcohol Dependence. 2020; 107854. [DOI:10.1016/j. drugalcdep.2020.107854] [PMID]

[52] García-García I, Morys F, Michaud A, Dagher A. Food Addiction, Skating on Thin Ice: A critical overview of neuroimaging findings. Food Addiction. 2020; 7:20-9. [DOI:10.1007/s40429-02000293-0]

[53] Murphy CM, MacKillop J. Food addiction and self-regulation. Compulsive Eating Behavior and Food Addiction. 2019; 193-216. [DOI:10.1016/B978-0-12-816207-1.00007-X] [PMID] [PMCID] 
[54] Tatsi E, Kamal A, Turvill A, Regina H. Emotion dysregulation and loneliness as predictors of food addiction. Journal of Health and Social Sciences. 2019; 4(1):43-58. [DOI:10.19204/2019/mtnd5]

[55] Wu S, Li X, Meng S, Fung T, Chan AT, Liang G, et al. Fruit and vegetable consumption, cigarette smoke, and leukocyte mitochondrial DNA copy number. American Journal Of Clinical Nutrition. 2019; 109(2):424-32. [DOI:10.1093/ajcn/nqy286] [PMID] [PMCID]

[56] Crossin R, Lawrence AJ, Andrews ZB, Duncan JR. Altered body weight associated with substance abuse: A look beyond food intake. Addiction Research \& Theory. 2019; 27(2):76-84. [DO I:10.1080/16066359.2018.1453064]

[57] Naish KR, Balodis IM. Reward processing in food addiction and overeating. Compulsive Eating Behavior and Food Addiction. 2019; 217-49. [DOI:10.1016/B978-0-12-816207-1.00008-1]

[58] Bbosa GS. Neurobiology of Substance of Abuse (Drugs) and Behavioural Addiction in Africa. Addiction in South and East Africa. 2019 ; 193-212. [DOI:10.1007/978-3-030-13593-5_12]

[59] Luisetto M, Almukhtar N, Mashori GR, Ahmadabadi BN, Sahu RK. Addiction and evolutionary process, common aspects in physio-pathologic pathways useful in pharmaco-toxicological approach. Advances in Clinical Toxicology. 2019; 4(1):000149. [DOI:10.23880/act-16000149]

[60] Becker JB, Chartoff E. Sex differences in neural mechanisms mediating reward and addiction. Neuropsychopharmacology. 2019; 44(1):166-83 [DOI:10.1038/s41386-018-0125-6] [PMID] [PMCID]

[61] Burleson JA, Kaminer Y. Self-efficacy as a predictor of treatment outcome in adolescent substance use disorders. Addictive Behaviors. 2005; 30(9):1751-64. [DOI:10.1016/j.addbeh.2005.07.006] [PMID]

[62] Gratz KL, Roemer L. Multidimensional assessment of emotion regulation and dysregulation: Development, factor structure, and initial validation of the difficulties in emotion regulation scale. Journal of Psychopathology and Behavioral Assessment. 2004; 26(1):41-54. [DOI:10.1023/B:JOBA.0000007455.08539.94]

[63] Orgeta V. Emotion dysregulation and anxiety in late adulthood. Journal of Anxiety Disorders. 2011; 25(8):1019-23. [DOI:10.1016/j. janxdis.2011.06.010] [PMID]

[64] Kun B, Demetrovics Z. Emotional intelligence and addictions: a systematic review. Substance Use \& Misuse. 2010; 45(7-8):1131-60. [DOI:10.3109/10826080903567855] [PMID]

[65] Brechan I, Kvalem IL. Relationship between body dissatisfaction and disordered eating: mediating role of self-esteem and depression. Eating Behaviors. 2015; 17:49-58. [DOI:10.1016/j.eatbeh.2014.12.008] [PMID]

[66] Frayn M, Sears CR, von Ranson KM. A sad mood increases attention to unhealthy food images in women with food addiction. Appetite. 2016; 100:55-63. [DOI:10.1016/j.appet.2016.02.008] [PMID]

[67] Albrecht U, Kirschner NE, Grüsser SM. [Diagnostic instruments for behavioural addiction: An overview (German)]. GMS Psycho-Social Medicine. 2007; 4. [PMID]

[68] Kurniasanti KS, Assandi P, Ismail RI, Nasrun MW, Wiguna T. Internet addiction: A new addiction? Medical Journal of Indonesia. 2019; 28(1):82-91. [DOI:10.13181/mji.v28i1.2752]
[69] Nene MJ, Gupta P. Cyberpsycho effect: A Critical study on the impact of internet addiction. InInternet and Technology Addiction: Breakthroughs in Research and Practice 2019 (pp. 488-500). IGI Global. [DOI:10.4018/978-1-5225-8900-6.ch028]

[70] Wong CK, Chen YM, Yen CF. Associations of parental bonding and adolescent internet addiction symptoms with depression and anxiety in parents of adolescents with attention deficit/hyperactivity disorder. Archives of Clinical Psychiatry (São Paulo). 2019; 46(2):40-3. [DOI:10.1590/0101-60830000000190]

[71] Abdolpour G, Shalchi B, Hamzezadeh S, Salehi A. [The mediating role of self-esteem on the relationship between emotional dysregulation and compassion with Internet addiction (Persian)]. Shenakht Journal of Psychology \& Psychiatry. 2019; 6(3):129-43. [DOI:10.29252/shenakht.6.3.129]

[72] Barrault S, Mathieu S, Brunault P, Varescon I. Does gambling type moderate the links between problem gambling, emotion regulation, anxiety, depression and gambling motives. International Gambling Studies. 2019; 19(1):54-68. [DOI:10.1080/144597 95.2018.1501403]

[73] Dochnal RB, Vetró Á, Kiss E, Baji I, Lefkovics E, Bylsma LM, Yaroslavsky I, Rottenberg J, Kovacs M, Kapornai K. Emotion regulation among adolescents with pediatric depression as a function of anxiety comorbidity. Frontiers in Psychiatry. 2019; 10:722. [DOI:10.3389/fpsyt.2019.00722] [PMID] [PMCID]

[74] Khantzian EJ. Psychodynamic psychotherapy for the treatment of substance use disorders. Textbook of Addiction Treatment: International Perspectives. 2015; 811-9. [DOI:10.1007/978-88-4705322-9_38] 